\title{
Novel sources of resistance to the soybean cyst nematode (Heterodera glycines) found in wild relatives of azuki bean (Vigna angularis) and their characteristics of resistance
}

\author{
A. Kushida $\cdot$ A. Tazawa $\cdot$ S. Aoyama $\cdot$ \\ N. Tomooka
}

Received: 23 April 2012/Accepted: 23 July 2012/Published online: 10 August 2012

(C) The Author(s) 2012. This article is published with open access at Springerlink.com

\begin{abstract}
Soybean cyst nematode (Heterodera glycines; $\mathrm{SCN}$ ) is becoming a serious problem in azuki bean (Vigna angularis var. angularis) production in Hokkaido, Japan. To find sources of resistance to SCN, we screened wild relatives of azuki bean and calculated "female indices" (FIs) of 342 accessions from 8 Vigna species that are cross-compatible with azuki bean. Twenty-three accessions belonging to $V$. hirtella, $V$. minima, $V$. nakashimae, $V$. riukiuensis, and
\end{abstract}

Electronic supplementary material The online version of this article (doi:10.1007/s10722-012-9895-6) contains supplementary material, which is available to authorized users.

A. Kushida

Memuro Upland Farming Research Station, NARO Hokkaido Agricultural Research Center, Shinsei-minami 9-4, Memuro-cho, Kasai-gun, Hokkaido 082-0071, Japan

\section{A. Tazawa}

Hokkaido Research Organization Tokachi Agricultural Experiment Station, Shinsei-minami 9-2, Memuro-cho,

Kasai-gun, Hokkaido 082-0081, Japan

\section{S. Aoyama}

Hokkaido Research Organization Kamikawa Agricultural

Experiment Station, Minami 1-5, Pippu-cho,

Kamikawa-gun, Hokkaido 079-0397, Japan

N. Tomooka $(\bowtie)$

Genetic Resources Center, National Institute

of Agrobiological Sciences, Kannon-dai 2-1-2,

Tsukuba, Ibaraki 305-8602, Japan

e-mail: tomooka@affrc.go.jp
$V$. tenuicaulis were resistant to the most prevalent SCN race, race 3 , but most of them were only moderately resistant or susceptible to race 5. Four promising accessions (V. minima JP205886, JP205891, and JP210806, and $V$. nakashimae JP107879) showed a high level of resistance to all SCN races found in Japan (1, 3, and 5) and race 2 (an experimentally derived race from race 5). Since the SCN-resistant soybean cultivars released in Japan are not resistant to races 2 and 5, these wild Vigna accessions may have resistance mechanisms different from that of soybean. Continuous culture of race 3 on promising accessions showed that it might be difficult to overcome the resistance of these accessions. The resistance sources found in this study will be useful in controlling SCN through the breeding of SCNresistant azuki bean cultivars. This study also showed the effectiveness of using wild genetic resources for identifying novel resistance sources.

Keywords Azuki bean - Resistance $\cdot$ Soybean cyst nematode $\cdot$ Vigna species

\section{Introduction}

Azuki bean (Vigna angularis (Willd.) Ohwi et Ohashi var. angularis) is one of the important legume crops in Hokkaido, which produces $90 \%$ of all Japanese azuki bean production. In 2010, the total area planted to azuki bean in Hokkaido was 23,800 ha, slightly more than the area planted to soybean (MAFF 2011). Azuki bean is a 
suitable host plant for the soybean cyst nematode ( $\mathrm{SCN}$ ), Heterodera glycines Ichinohe, and permits copious reproduction of SCN (Ichinohe 1953; Ozaki and Asai 1963; Watanabe and Saito 1993). However, as yield losses of azuki bean caused by SCN are not as devastating as those of soybean, little effort has been made to control SCN in azuki bean. Recently, however, damage has started to increase because the population density of $\mathrm{SCN}$ in Hokkaido fields has increased owing to the short-term rotation of legumes (Aoyama and Kushida 2011). Consequently, the yield loss of soybean, which is one of the rotation crops, is increasing. Thus, controlling $\mathrm{SCN}$ in azuki bean production is becoming increasingly important. Although the use of resistant cultivars is an effective practice for managing SCN, genetic sources of resistance to SCN have not yet been identified in azuki bean.

The numbers of female SCN on the roots of about 30 azuki bean cultivars were investigated and compared at the Tokachi Agricultural Experiment Station, but no cultivars with few females were found (unpublished data). Shimizu and Mitsui (1987) reported differences in the rate of SCN reproduction among some azuki bean cultivars, but cultivars with a low rate were not sufficiently resistant to control the SCN population. In contrast, we recently identified an accession (JP235878) of Vigna riukiuensis with a very low female index (FI; see Materials and Methods for definition) value (Aoyama and Kushida 2011). This accession proved to be resistant to a number of diseases, including brown stem rot, phytophthora stem rot, and fusarium wilt, and it was crossed with azuki bean to introduce these resistance genes in the Tokachi Agricultural Experiment Station. The SCN resistance (low FI) of this accession was inherited in some derivative lines (Aoyama and Kushida 2011). Since other genetic resources of resistance are likely to be present among other wild relatives of azuki bean, we investigated these relatives. The objectives of this study were to identify sources of resistance to SCN by screening a large number of accessions of Vigna species that are cross-compatible with azuki bean, and to determine the characteristics of the resistance.

\section{Materials and methods}

Plant materials

We screened 342 accessions from 8 Vigna species for $\mathrm{SCN}$ resistance: Vigna angularis (Willd.) Ohwi et
Ohashi var. nipponensis (Ohwi) Ohwi et Ohashi (112 accessions), V. hirtella Ridley (23), V. minima (Roxb.) Ohwi et Ohashi (25), V. nakashimae (Ohwi) Ohwi et Ohashi (31), V. nepalensis Y. Tateishi et N. Maxted (6), V. riukiuensis (Ohwi) Ohwi et Ohashi (27), $V$. tenuicaulis N. Tomooka et N. Maxted (28), and V. umbellata (Thunb.) Ohwi et Ohashi (90). The passport information of these accessions is provided in Supplementary Table 1. These wild Vigna species are cross-compatible with azuki bean and are primarily distributed in Southeast and East Asia (Tomooka et al. 2002). The accessions were provided by the Genebank of the National Institute of Agrobiological Sciences (NIAS) in Tsukuba, Japan.

Nematode populations

Three races of SCN (races 1, 3, and 5) were collected from fields in Hokkaido, and one race (race 2) was experimentally obtained from continuous culture of race 5 on the resistant soybean cultivar 'Suzuhime'. Race 3 was maintained on the susceptible soybean cultivar 'Kitamusume'. Since races 1 and 5 are more virulent than race 3 , and so seriously damages 'Kitamusume' that they could not increase their population size on 'Kitamusume'. Therefore, races 1 and 5 were maintained on the resistant soybean cultivar 'Toyomusume', which shows a suitable level of resistance to enable the population size of these races to increase sufficiently. The populations were maintained at $4{ }^{\circ} \mathrm{C}$.

Calculation of the female index (FI)

Two seeds from each accession were sown in a pot (9$\mathrm{cm}$ diameter and 7.5-cm height) filled with approximately $250 \mathrm{~mL}$ of autoclaved soil and maintained in a greenhouse at $20-25{ }^{\circ} \mathrm{C}$. After germination, seedlings were reduced to 1 per pot. When each seedling had produced 5 or 6 foliage leaves, 1,000 second-stage juveniles (J2) were inoculated into each pot. J2s were prepared as follows. Cysts of SCN were isolated from soil using a method described by Yoshihara and Kegasawa (1989) and crushed to release eggs. Eggs suspended in water were passed through a sieve with a pore size of $64 \mu \mathrm{m}$ to remove debris. The eggs were rinsed 3 times with distilled water and resuspended in distilled water and were then incubated at $25{ }^{\circ} \mathrm{C}$ for 1 week. Then they were suspended in root leachate 
obtained from kidney bean seedlings according to the method described by Kushida et al. (2003) and incubated at $27{ }^{\circ} \mathrm{C}$. After 1 week, hatched J2s were collected by the Baermann method.

At 30-35 days after $\mathbf{J} 2$ inoculation, we dislodged SCNs from the soil and roots by rubbing the roots gently in water, then collected them on a $250-\mu \mathrm{m}$-pore sieve and counted the females under a stereomicroscope. The wet roots were patted dry with a paper towel and weighed.

The azuki bean cultivar 'Erimo-shozu' was grown as a control. 'Erimo-shozu' is the most popular azuki bean cultivar in Hokkaido and is highly susceptible to all SCN races. The resistance of each accession was evaluated as the FI, which was calculated as (average number of females on test accession/average number of females on 'Erimo-shozu') $\times 100$.

First and second screenings

In the first screening, FIs of all accessions against SCN race 3 were measured with no replication. Accessions with FI $\leq 25$ were selected and used in the second screening, in which FIs against SCN races 3 and 5 were determined with 1-3 replications. An accession of $V$. riukiuensis, JP235878, which showed a low FI against SCN (Aoyama and Kushida 2011), was included in the second screening.

Testing of resistance of selected accessions to SCN races $1,2,3$, and 5

We used 4 SCN-resistant accessions ( $V$. minima JP205886, JP205891, JP210806, and V. nakashimae JP107879) from the second screening and 2 susceptible accessions ( $V$. minima JP210821 and $V$. nakashimae JP212341) from the first screening showing vigorous growth to investigate FIs against $\mathrm{SCN}$ races 1, 2, 3, and 5 with 4 replications. Differences among FIs against each SCN race were analyzed by the Kruskal-Wallis test followed by Scheffé's multiple comparison test. Values were $\log$-transformed $\left(\log _{10}[\mathrm{FI}+1]\right)$ for analysis.

\section{Effect of continuous culture of $\mathrm{SCN}$ race 3}

To investigate whether the resistance of selected Vigna accessions could be overcome, we continuously cultured SCN race 3 on the roots of resistant accessions. Two resistant accessions ( $V$. minima JP205891 and V. nakashimae JP107879) were planted in plastic pots (16-cm diameter and 20-cm height). When each seedling had produced 5 to 8 leaves, we inoculated approximately $8,000 \mathrm{~J} 2 \mathrm{~s}$ of race 3 into each pot. Plants were maintained in a greenhouse for 4 months. Cysts were isolated from the soil and used to prepare $\mathrm{J} 2$ inoculum for the next culture. After a second culture, all cysts in the pot were isolated and counted. J2s that had hatched were gathered and inoculated onto 5 replicated seedlings of the 2 resistant accessions, the 2 susceptible control accessions, and 'Erimo-shozu', and FIs were calculated as above. FIs of the original population, which had not been cultured on resistant accessions, were also examined at the same time and compared. To analyze the differences in FIs between the original population and the cultured population, we compared log-transformed FIs with the Mann-Whitney $U$ test.

Development of juveniles in the roots of resistant and susceptible accessions

Seeds of the 2 selected resistant and 2 selected susceptible accessions were sown in 16 pots each and grown as in the first screening. When each seedling had produced 3 or 4 leaves, approximately $500 \mathrm{~J} 2 \mathrm{~s}$ of race 5 were inoculated into each pot, and plants were maintained in a greenhouse $\left(22-26^{\circ} \mathrm{C}\right)$. Seedlings from 4 pots were harvested 3,14 , and 21 days after inoculation, and soil was gently washed off the root systems with tap water. Roots were soaked in $3 \%$ formaldehyde solution for a few days at room temperature, washed in tap water, and stained by boiling in lactoglycerol containing $0.05 \%$ acid fuchsine (Hooper 1986). On day 21, females that came out from the roots during the washing process were collected with a $212-\mu \mathrm{m}$-pore sieve and counted. Stained roots were homogenized in $30-50 \mathrm{~mL}$ of distilled water, and the $\mathrm{J} 2 \mathrm{~s}, \mathrm{~J} 3 \mathrm{~s}$, J4s, and adult females in $1 \mathrm{~mL}$ of homogenate were counted in 5 replicates. The sex of $\mathrm{J} 4 \mathrm{~s}$ was determined from the internal form or shape (shown later in Fig. 5). J2s and J3s were discriminated by tail form. J3s and J4s (female) were roughly discriminated by size. On day 35 , females were collected from the soil and roots, as described above, and counted. Differences in the numbers of nematodes of each stage between the resistant and 


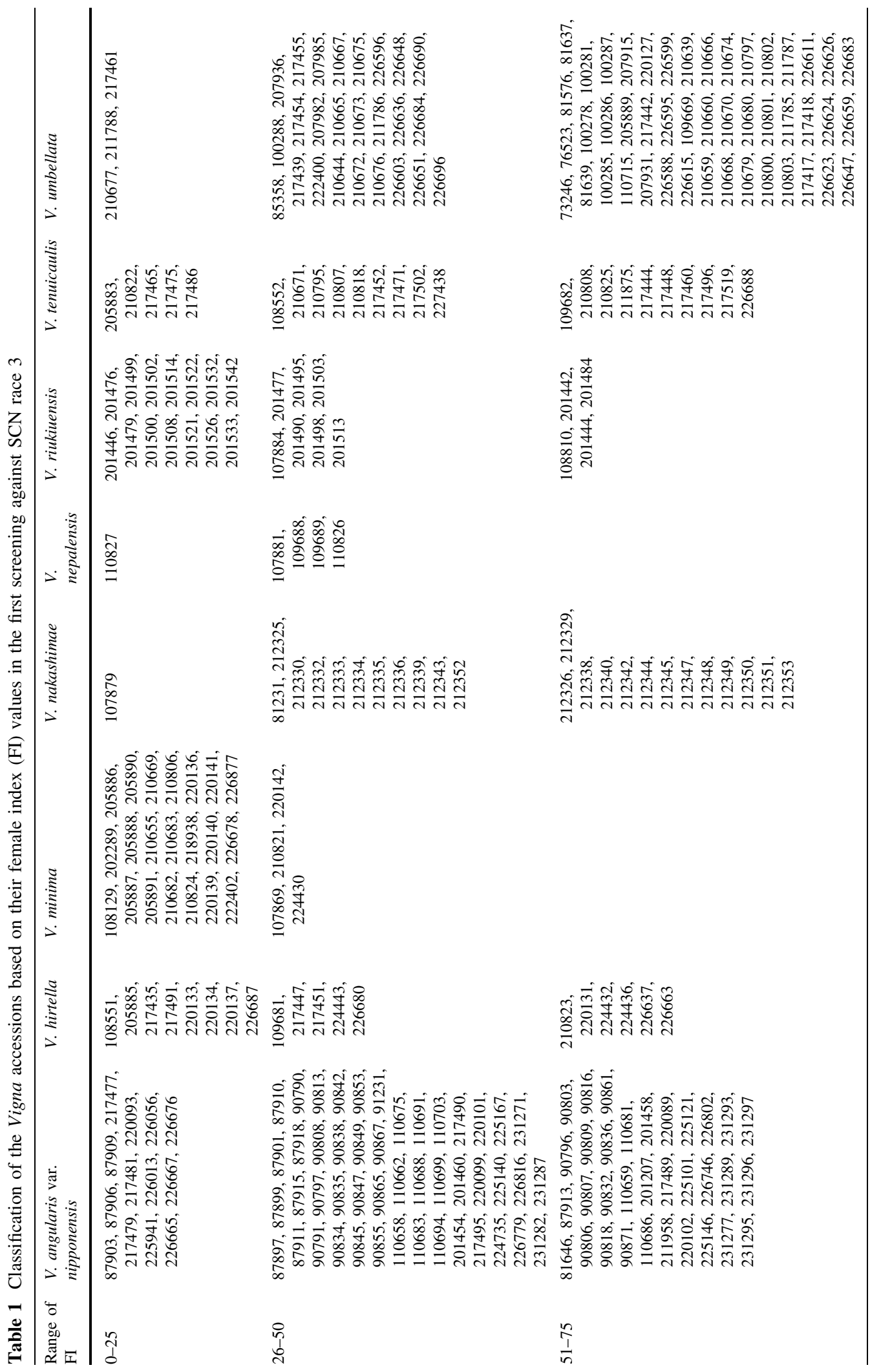




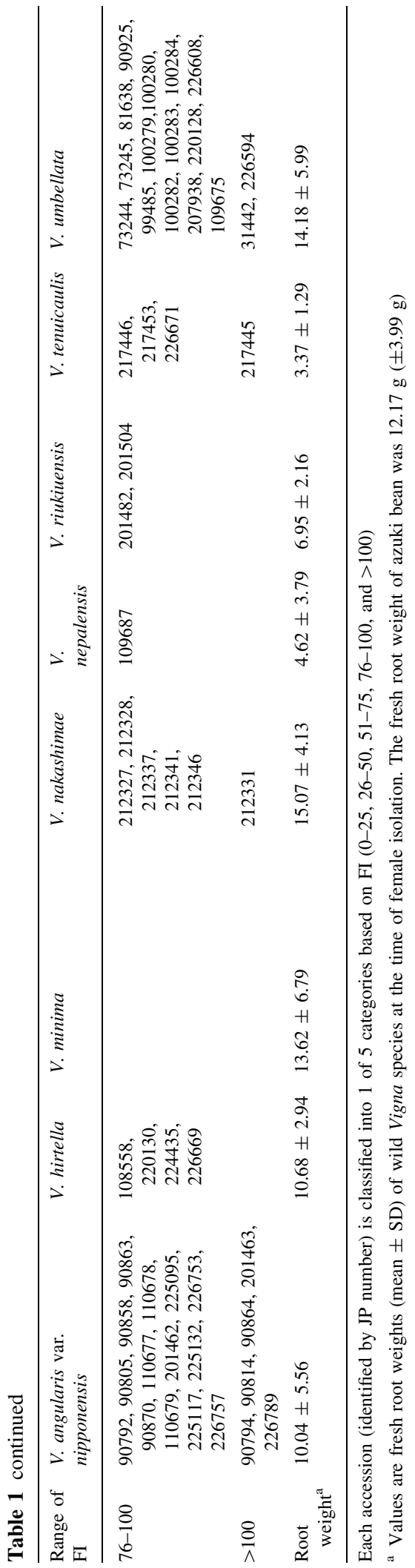

susceptible accessions were compared by MannWhitney $U$ test.

\section{Results}

First screening

Each accession was classified into 1 of 5 FI categories (0-25, 26-50, 51-75, 76-100, and $>100$; Table 1). FIs of tested accessions ranged between 3 and 121. All accessions had females. Low FIs were common among $V$. minima accessions. High FIs were generally common among $V$. umbellata and $V$. nakashimae accessions. All V. nepalensis accessions showed very poor growth. A positive correlation between FI and fresh root weight $(P<0.05$, Spearman's rank correlation analysis) suggested that the low FIs of $V$. nepalensis might have been caused by poor growth rather than by resistance. Because it was difficult to accurately judge the resistance of these accessions, we omitted them from the second screening. Although the root weights of $V$. tenuicaulis were also small, there was no correlation between FI and root weight in this species. Therefore, we used accessions of $V$. tenuicaulis in the second screening. We re-examined 65 accessions with FI $\leq 25$ in the second screening.

Second screening

Vigna hirtella JP108551 and V. tenuicaulis JP205883 were omitted owing to their very poor growth. Accessions with $\mathrm{FI} \leq 10$ against $\mathrm{SCN}$ race 3 were found in V. hirtella, V. minima, V. nakashimae, V. riukiuensis, and $V$. tenuicaulis, but most of them showed FI $>10$ against race 5 (Table 2). The FIs of 5 accessions of $V$. minima and 1 of $V$. nakashimae against race 5 were $\leq 10$. Since we considered the importance of the resistance against SCN race 5 which can grow normally on SCNresistant soybean, we selected these 6 accessions first. From these accessions, we selected 3 accessions of $V$. minima (JP205886, JP205891, JP210806) and 1 of $V$. nakashimae (JP107879) on the basis of their resistance to $\mathrm{SCN}$ race 3 and the vigor of the plants.

FIs against races 3 and 5 on $V$. riukiuensis JP235878, which was found to be resistant to SCN in the previous study, were around 30. Many other accessions of $V$. riukiuensis showed lower FIs than JP235878. 
Table 2 Female index (FI) values on each accession of Vigna against SCN races 3 and 5

\begin{tabular}{|c|c|c|c|c|c|c|c|c|}
\hline \multirow[t]{2}{*}{ JP no. } & \multicolumn{2}{|c|}{ Population } & \multirow[t]{2}{*}{ JP no. } & \multicolumn{2}{|c|}{ Population } & \multirow[t]{2}{*}{ JP no. } & \multicolumn{2}{|c|}{ Population } \\
\hline & Race 3 & Race 5 & & Race 3 & Race 5 & & Race 3 & Race 5 \\
\hline \multicolumn{3}{|c|}{$V$. angularis var. nipponensis } & \multicolumn{3}{|c|}{$V$. minima } & \multicolumn{3}{|l|}{ V. riukiuensis } \\
\hline 87903 & $67(2)$ & $89(2)$ & 108129 & $16(1)$ & $10(3)$ & 201446 & $11(2)$ & $47(3)$ \\
\hline 87906 & $78(1)$ & $69(2)$ & 202289 & $5(1)$ & $7(3)$ & 201476 & $12(2)$ & $29(3)$ \\
\hline 87909 & $45(1)$ & $72(2)$ & 205886 & $14(1)$ & $8(3)$ & 201479 & $10(2)$ & $13(3)$ \\
\hline 217477 & $24(3)$ & $54(2)$ & 205887 & $40(1)$ & $51(3)$ & 201499 & $6(2)$ & $17(3)$ \\
\hline 217479 & $114(4)$ & $111(2)$ & 205888 & $48(1)$ & $33(3)$ & 201500 & $8(2)$ & $30(3)$ \\
\hline 217481 & $69(4)$ & $86(2)$ & 205890 & $25(1)$ & $23(3)$ & 201502 & $13(2)$ & $13(3)$ \\
\hline 220093 & $89(4)$ & $101(2)$ & 205891 & $10(1)$ & $4(3)$ & 201508 & $6(2)$ & $29(3)$ \\
\hline 225941 & $71(3)$ & $79(2)$ & 210655 & $8(1)$ & $54(3)$ & 201514 & $2(2)$ & $24(3)$ \\
\hline 226013 & $18(4)$ & $58(2)$ & 210669 & $38(1)$ & $29(3)$ & 201521 & $7(1)$ & $44(3)$ \\
\hline 226056 & $80(4)$ & $84(2)$ & 210682 & $52(1)$ & 77 (3) & 201522 & $6(2)$ & $24(3)$ \\
\hline 226665 & $72(4)$ & $79(2)$ & 210683 & $20(1)$ & $35(3)$ & 201526 & $5(2)$ & $39(3)$ \\
\hline 226667 & $52(4)$ & $72(2)$ & 210806 & $8(1)$ & $6(3)$ & 201532 & $2(2)$ & $34(3)$ \\
\hline \multirow[t]{2}{*}{226676} & $38(4)$ & $65(2)$ & 210824 & $14(1)$ & $33(3)$ & 201533 & $6(2)$ & $54(3)$ \\
\hline & & & 220136 & $94(1)$ & $43(3)$ & 201542 & $7(2)$ & $25(3)$ \\
\hline V. hirtella & & & 220139 & $48(1)$ & $43(3)$ & 235878 & $27(2)$ & $34(3)$ \\
\hline 205885 & $16(3)$ & $48(3)$ & 220140 & $2(1)$ & $95(2)$ & & & \\
\hline 217435 & $23(3)$ & $85(3)$ & 220141 & $28(1)$ & $65(3)$ & \multicolumn{3}{|l|}{ V. tenuicaulis } \\
\hline 217491 & $9(3)$ & $39(3)$ & 222402 & $7(1)$ & $19(3)$ & 210822 & $1(2)$ & $48(3)$ \\
\hline 220133 & $10(3)$ & $53(3)$ & 226678 & $38(1)$ & $55(3)$ & 217465 & $44(4)$ & $60(3)$ \\
\hline 220134 & $40(3)$ & $84(3)$ & 218938 & $9(1)$ & $21(3)$ & 217475 & $25(4)$ & $44(3)$ \\
\hline 220137 & $44(3)$ & $93(3)$ & 226877 & $1(1)$ & $14(3)$ & 217486 & $22(3)$ & $34(3)$ \\
\hline \multirow[t]{5}{*}{226687} & $13(3)$ & $62(3)$ & & & & & & \\
\hline & & & \multicolumn{3}{|c|}{ V. nakashimae } & \multicolumn{3}{|l|}{ V. umbellata } \\
\hline & & & 107879 & $5(2)$ & $10(3)$ & 210677 & 77 (3) & $76(3)$ \\
\hline & & & & & & 211788 & $70(3)$ & $104(3)$ \\
\hline & & & & & & 217461 & $50(3)$ & 77 (2) \\
\hline
\end{tabular}

Values in parentheses show the numbers of tested plants

Testing of resistance to various SCN races

The susceptible V. minima JP210821 and V. nakashimae JP212341 showed high FIs against all 4 SCN races. The mean FIs of all resistant accessions against each SCN race were $<10$, except for that of $V$. minima JP205886 against race 1 (Fig. 1). In particular, $V$. minima JP205891 and JP210806 showed high resistance to races 2 and 5, which can break down the SCN resistance of soybean cultivars in Japan. Although all FIs were low, those of $V$. minima JP205886 showed significant differences between races 1 and 3 , and those of $V$. minima JP2 10806 showed significant differences between race 3 and all other races $(P<0.05)$.
Effects of continuous culture of SCN on resistant Vigna accessions

Two SCN resistant accessions, V. minima JP205891 and V. nakashimae JP107879, were used for continuous SCN culturing. After the second cycle of cultivation, we isolated 212 cysts from the pot of $V$. minima JP205891 and 2,282 cysts from V. nakashimae JP107879. These numbers are both lower than the number of J2s inoculated (ca. 8,000). The FIs of the resistant accessions against the $\mathrm{SCN}$ populations raised on resistant accessions were very low and were not significantly different from the FIs against the original SCN populations $(P>0.05$, Fig. 2$)$. In 
Fig. 1 Female index (FI) values of each accession of wild Vigna species against 4 $\mathrm{SCN}$ races. Error bars indicate standard deviations

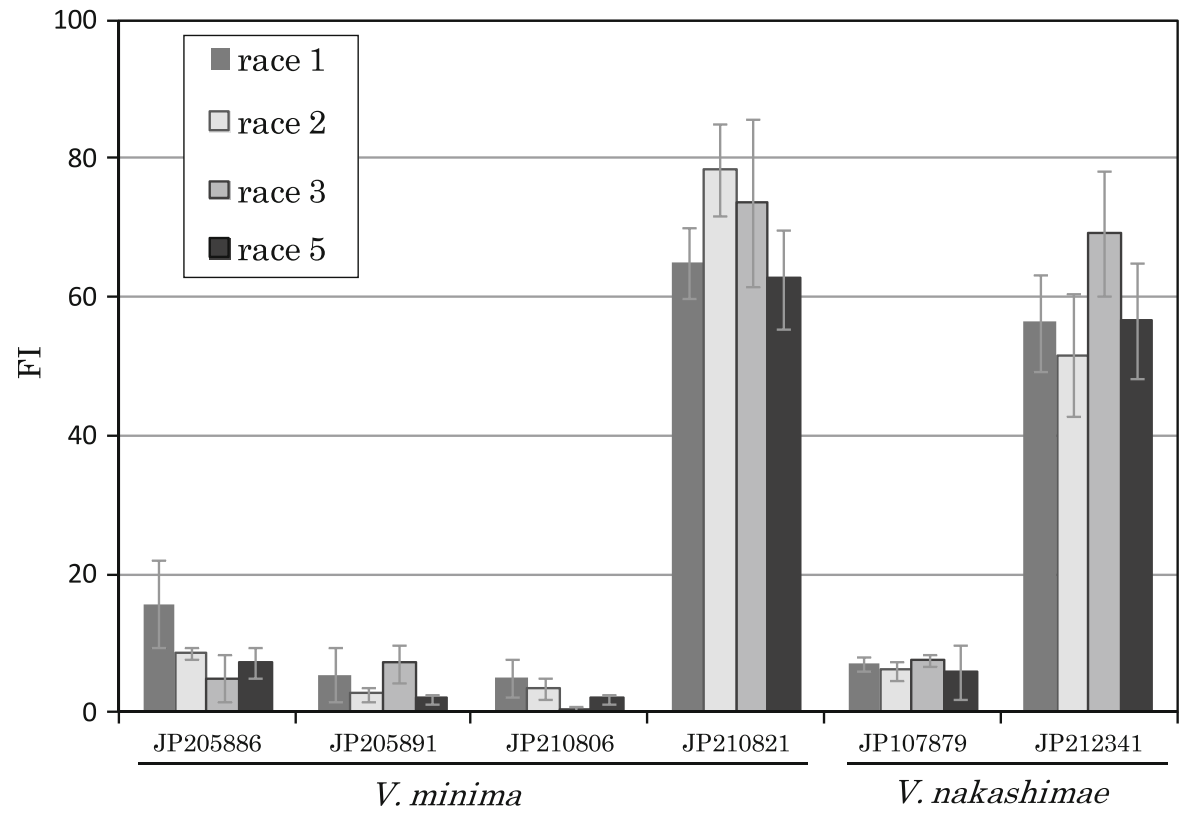

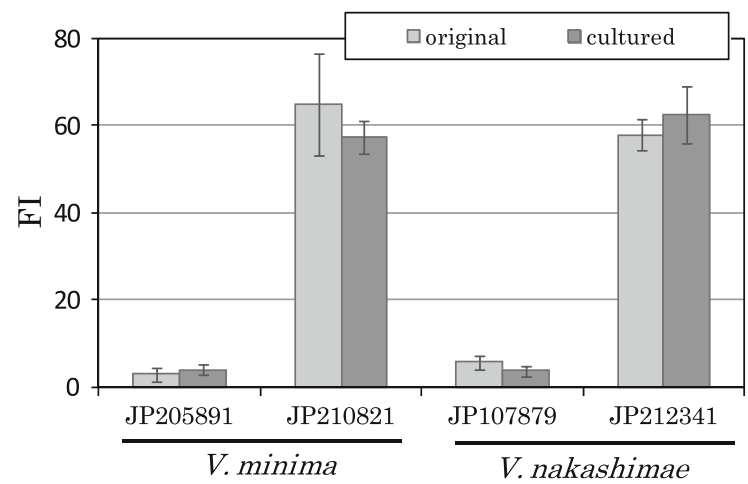

Fig. 2 Female index (FI) values of each accession against original and cultured SCN. Values are means of 5 replications; error bars indicate standard deviation. There were no significant differences between FIs against the original and cultured populations $(P>0.05$, Mann-Whitney $U$ test, $n=5)$

contrast, the FIs of the 2 susceptible accessions against both cultured and original SCN were very high.

Development of SCN juveniles on the roots of resistant and susceptible accessions

Only J2s were isolated from the roots of each accession 3 days after inoculation of $\mathbf{J} 2 \mathrm{~s}$. The mean number of $\mathrm{J} 2 \mathrm{~s}$ in the roots of resistant accessions was 360-400, not significantly different from the number on susceptible accessions (data not shown). The mean numbers of SCN juveniles of each stage that were isolated from the roots of each accession on 14 and 21 days after J2s inoculation were shown in Figs. 3 and 4, respectively. $\mathrm{J} 2 \mathrm{~s}, \mathrm{~J} 3 \mathrm{~s}$, and $\mathrm{J} 4 \mathrm{~s}$ were isolated from the roots of all accessions 14 days after inoculation. Both fat J4s (thought to be females) and thin J4s (males, visible adult male in their body) were present (Fig. 5). There were significant differences between resistant and susceptible accessions of $V$. minima in all stages, and between those of $V$. nakashimae in female $\mathrm{J} 4 \mathrm{~s}$ (Fig. 3). The mean number of female J4s collected from resistant accessions was significantly lower than those collected from susceptible accessions, whereas the number of $\mathbf{J} 2 \mathrm{~s}$ collected from resistant accessions was higher (significantly in V. minima). Most of J2s were not stained deeply. J3s stained well, but some were shrunken (Fig. 5).

$\mathrm{J} 2 \mathrm{~s}, \mathrm{~J} 3 \mathrm{~s}, \mathrm{~J} 4 \mathrm{~s}$, and adults were isolated from the roots of all accessions 21 days after inoculation (Fig. 4). J2s and most of $\mathrm{J} 3 \mathrm{~s}$ were transparent. The numbers of females (adult $+\mathrm{J} 4$ ) on each accession at 21 days were the same as or slightly higher than the numbers of J4 females at 14 days (Figs. 3 and 4). Moreover, these values were similar to the numbers isolated at 35 days (data not shown). On the susceptible accessions, the numbers of males were nearly identical to or slightly lower than the numbers of females at 14 and 21 days. On the resistant accessions, in contrast, the numbers of males were significantly more than the number of females at both days $(P<0.05)$. 


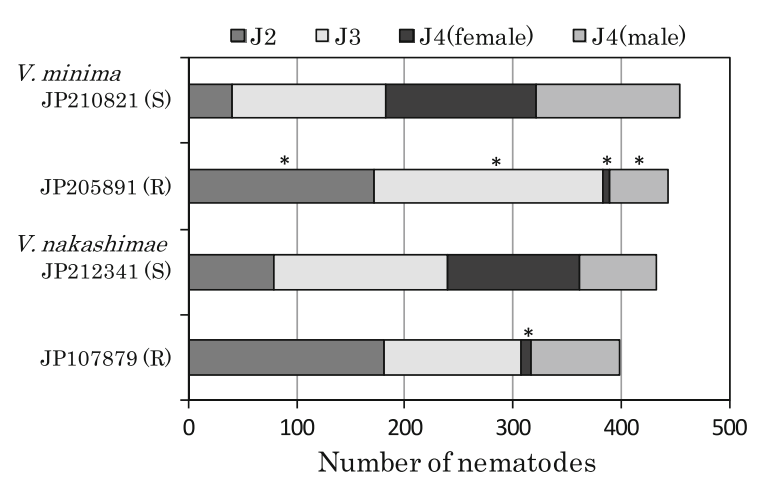

Fig. 3 Mean numbers of SCN juveniles of each stage that were isolated from the roots of each accession 14 days after inoculation of 500 second-stage juveniles $(n=4)$. (R): resistant accession, (S): susceptible accession. *Mean of resistant accession is significantly different from that of the control accession of the same species $(P<0.05$, Mann-Whitney $U$ test, $n=4)$

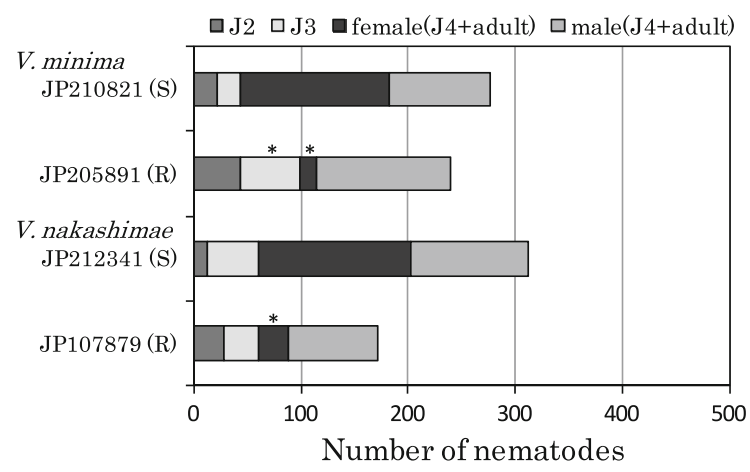

Fig. 4 Mean numbers of SCN juveniles of each stage that were isolated from the roots of each accession 21 days after inoculation of 500 second-stage juveniles $(n=4)$. *Mean of resistant accession is significantly different from that of the control accession of the same species $(P<0.05$, MannWhitney $U$ test, $n=4$ )

\section{Discussion}

Since all Vigna species investigated held accessions with FIs as high as that of 'Erimo-shozu', these wild species might be suitable hosts for SCN (Table 1). Since $V$. angularis var. nipponensis and V. nakashimae are distributed in northeastern China, Korea, and Japan (Tomooka et al. 2002), where SCN is also distributed, SCN may be maintained on these plants outside of cultivated fields. Although most $V$. minima accessions had low FIs, this species was ranked as a host, because JP210821, which we used as a susceptible control, consistently showed high FIs.

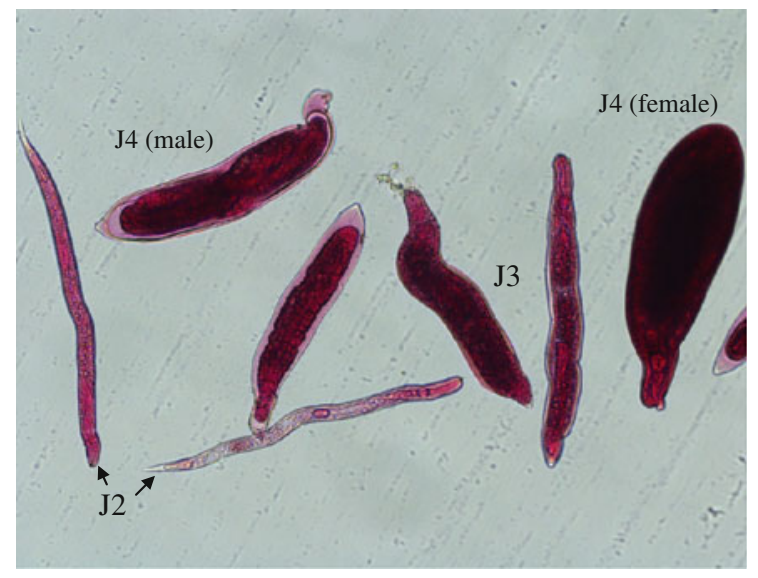

Fig. 5 Juveniles isolated from the roots of V. minima JP205891 14 days after $\mathbf{J} 2$ inoculation

Plants with FI $<10$ are considered to be resistant to SCN, and those with FIs between 10 and 30 to be moderately resistant (Schmitt and Shannon 1992). In the first screening, we selected accessions with $\mathrm{FI} \leq 25$ as resistant candidates because we tested them without replication. In the second screening, we determined accessions with $\mathrm{FI} \leq 10$ to be resistant to SCN.

Accessions with $\mathrm{FI} \leq 10$ against race 3 were found in $V$. hirtella, V. minima, V. nakashimae, V. riukiuensis, and V. tenuicaulis (Table 2). These accessions were classified to be resistant to $\mathrm{SCN}$ race $3 . V$. riukiuensis JP235878, the first reported resistant resource (Aoyama and Kushida 2011), was only moderately resistant $(\mathrm{FI} \approx 30$ ). Therefore, the accessions we identified here are the first with a high level of $\mathrm{SCN}$ resistance. As we found resistant accessions in 5 species, sources of SCN resistance may be widespread among Vigna species.

Resistant accessions of $V$. minima, $V$. hirtella, and V. riukiuensis were collected primarily from Southeast Asia (Thailand, Myanmar, Laos, and Okinawa-Japan), where SCN is not present. In fact, no species of cyst nematodes that parasitize beans have been found in these regions (Evans and Rowe 1998). Therefore, these accessions might have developed resistance to other pests that might coincidentally work against SCN.

Only 1 among $31 \mathrm{~V}$. nakashimae accessions (JP107879) was found to be resistant. This accession was collected in the Goto Islands in Nagasaki Prefecture in Japan, whereas the other 30 were collected in 
Korea. Therefore, the SCN resistance of this accession might have developed after geographic isolation. In contrast, many $V$. minima accessions had low FIs, suggesting that SCN resistance is widespread among this species. Three promising resistant accessions (JP205886, JP205891, and JP210806) were selected. These accessions were collected in northern Thailand (Nan, alt. $625 \mathrm{~m}$ ), northeastern Thailand (Surin, $305 \mathrm{~m}$ ), and central Myanmar (Kalaw, 1,230 m), respectively. Susceptible $V$. minima accessions selected in the first screening (JP107869, JP210821, JP220142, and JP224430) were collected in central Thailand (Uthai Tani, alt. $100 \mathrm{~m}$ ), southern Myanmar (Setse, $5 \mathrm{~m}$ ), southern Laos (Pakse, $100 \mathrm{~m}$ ), northern Laos (Udom Xai, $949 \mathrm{~m}$ ), and northern Thailand (Nan, 1,400 m), respectively. There was no clear pattern of geographic distribution of resistant or susceptible accessions.

SCN races 1, 3, and 5 are confirmed to be distributed in Japan (Inagaki 1979; Shimizu 1987). Race 2 has not been found in Japan. However, it may appear in the future through the continuous cultivation of resistant soybean cultivars, as it was experimentally selected by the continuous culture of race 5 on SCNresistant soybean 'Suzuhime'. Race 3, which is distributed the most widely in Japan, can seldom reproduce on the resistant soybean cultivars grown in Japan. However, races 1, 5, and 2 can reproduce on resistant soybeans, with the ability to reproduce increasing in that order. Race 2 develops well on the roots of the strongest resistant soybean cultivar in Japan (A. Kushida, unpublished). Many accessions showing resistance to race 3 showed moderate or no resistance to race 5 (Table 2). Therefore, the mechanisms of resistance in these Vigna accessions are similar to those of soybean. On the other hand, four accessions ( $V$. minima JP205886, JP205891, and JP210806, and V. nakashimae JP107879) selected from the screening tests showed high levels of resistance to all SCN races in Japan (1, 3, and 5) and race 2. Therefore, the resistance of these Vigna accessions may be more effective for controlling SCN than that of resistant soybean cultivars and may have a unique resistance mechanism.

Field populations of SCN are mixtures of parasitic phenotypes. Therefore, continuous planting of resistant soybean cultivars results in the selection of resistance-breaking SCN races and the loss of resistance (Niblack 1992; Riggs and Schuster 1998; Young 1992). Young (1994) cultured SCN race 5 on the soybean cultivar 'Cordell' for 10-14 generations and confirmed that the FIs increased from 10 to 77 and that the race changed to race 14 . To test whether the resistance of $V$. minima JP205891 and V. nakashimae JP107879 could also be broken down by continuous cultivation, we cultured SCN race 3 on them twice for 4 months each. The FIs did not change significantly (Fig. 2). Very few cysts compared to the number of inoculated $\mathbf{J} 2(8,000)$ were isolated after the second culture, but we observed some white females on the roots and cysts with many egg shells at the end of second culture. These clues indicate that SCN underwent several generations on the resistant Vigna accessions. In contrast, previous experiments showed that the mean FIs of the same race 3 population that we used here increased significantly after continuous culture on 2 crops of resistant soybeans (A. Kushida, unpublished). Therefore, our results suggest that it might be difficult to overcome the resistance of these promising Vigna accessions. However, it will be necessary to culture SCN on them over several crops before we can be sure.

The number of $\mathrm{J} 2 \mathrm{~s}$ invading the roots of resistant soybeans was not different from the number invading susceptible cultivars, so the resistance was considered to start working after their invasion into the roots (Huang 1998). Similarly, the number of J2s invading the roots of the resistant Vigna accession was not significantly different from the number invading the susceptible accession. Therefore, this resistance might also begin to work after invasion. Many J2s were still present on day 14 after inoculation of resistant Vigna accessions (Fig. 3), but most of them were thought to be dead because they were not stained deeply, indicating that they had failed to parasitize to the roots. The number of J2s decreased on day 21 (Fig. 4), maybe because their transparency made them hard to see. Although some juveniles developed to J4 or higher on the roots of resistant accessions, most of these were male (Figs. 3,4). Similar phenomena have been observed in resistant soybean cultivars (Colgrove and Niblack 2005; Luedders 1987; Ross 1958; Sato and Omori 1969; Yuhara and Inagaki 1963). Since the resistance of $V$. minima JP205891 and $V$. nakashimae JP107879 was effective against all the SCN races tested, the mechanism of this resistance might be different from that of soybean resistance. However, no clear differences in the developmental pattern of SCN were evident between resistant soybean cultivars and 
resistant Vigna accessions. To clarify the characteristics of the Vigna accessions' resistance, it will be necessary to perform a histological survey of the syncytium in detail.

We found promising genetic resources with resistance to all races of SCN present in Japan in wild Vigna species. These resources will find use in the breeding of azuki bean cultivars that are resistant to SCN.

Acknowledgments This study was supported by the National Institute of Agrobiological Sciences (NIAS) Genebank project.

Open Access This article is distributed under the terms of the Creative Commons Attribution License which permits any use, distribution, and reproduction in any medium, provided the original author(s) and the source are credited.

\section{References}

Aoyama S, Kushida A (2011) Breeding soybean cyst nematode resistant azuki bean using wild Vigna germplasm. Proceedings of 14th NIAS international workshop on genetic resources, pp 103-108. http://www.gene.affrc.go.jp/pdf/ misc/international-WS_14_103.pdf

Colgrove AL, Niblack TL (2005) The effect of resistant soybean on male and female development and adult sex ratios of Heterodera glycines. J Nematol 37:161-167

Evans K, Rowe JA (1998) Distribution and economic importance. In: Sharma SB (ed) The cyst nematodes. Kluwer Academic Publishers, London, pp 1-30

Hooper DJ (1986) Preserving and staining nematodes in plant tissues. In: Southey JF (ed) Laboratory methods for work with plant and soil nematodes. HMSO, London, pp 81-85

Huang JS (1998) Mechanisms of resistance. In: Sharma SB (ed) The cyst nematodes. Kluwer Academic Publishers, London, pp 353-368

Ichinohe M (1953) On the parasitism of soybean cyst nematode, Heterodera glycines. Res Bull Hokkaido Natl Agric Exp Stn 64:113-124

Inagaki H (1979) Race status of five Japanese populations of Heterodera glycines. Jpn J Nematol 9:1-4

Kushida A, Suwa N, Ueda Y, Momota Y (2003) Effects of Crotalaria juncea and C. spectabilis on hatching and population density of the soybean cyst nematode, Heterodera glycines (Tylenchida: Heteroderidae). Appl Entomol Zool 38:393-399

Luedders VD (1987) Selection against Heterodera glycines males by soybean lines with genes for resistance. J Nematol 19:459-462
MAFF (2011) Agricultural land (pulses and miscellaneous cereals) In: 85th statistical yearbook of ministry of agriculture, forestry and fisheries (2009-2010). http://www.maff. go.jp/e/tokei/kikaku/nenji_e/85nenji/index.html. Accessed 28 Nov 2011 (in Japanese)

Niblack TL (1992) The race concept. In: Riggs RD, Wrather JA (eds) Biology and management of the soybean cyst nematode. APS Press, St Paul, pp 73-86

Ozaki K, Asai M (1963) Studies on the rotation systems, 2. The relationships between crop sequence and the soybean cyst nematode population in the soil. Res Bull Hokkaido Natl Agric Exp Stn 81:11-21 (in Japanese with English summary)

Riggs RD, Schuster RP (1998) Management. In: Sharma SB (ed) The cyst nematodes. Kluwer Academic Publishers, London, pp 388-416

Ross JP (1958) Host-parasite relationship of the soybean cyst nematode in resistant soybean roots. Phytopathology 48: $578-579$

Sato T, Omori H (1969) Studies on the control of the soybean cyst nematode, Heterodera glycines Ichinohe. Res Bull Iwate Pref Agric Exp Stn 13:1-47 (in Japanese with English summary)

Schmitt DP, Shannon G (1992) Differentiating soybean cyst nematode race and resistance response of soybean. Crop Sci 32:275-277

Shimizu K (1987) Races of the soybean cyst nematode in Hokkaido. Plant Prot 41:117-123 (in Japanese)

Shimizu K, Mitsui Y (1987) Varietal difference of azuki bean and kidney bean in susceptibility to the race of the soybean cyst nematode, Heterodera glycines Ichinohe, 1952. Ann Rep Soc Plant Prot N Jpn 38:109-110 (in Japanese with English summary)

Tomooka N, Vaughan DA, Maxted N, Moss H (2002) The Asian Vigna. Genus Vigna subgenus Ceratotropis genetic resources. Kluwer Academic Publishers, London

Watanabe M, Saito K (1993) Ecological control of Heterodera glycines injurious to azuki bean. Bull Tochigi Pref Agric Exp Stn 40:47-58 (in Japanese with English summary)

Yoshihara T, Kegasawa K (1989) A method for the extraction of cyst of soybean cyst nematode from large volumes of wet soil. Jpn J Nematol 19:52-55 (in Japanese)

Young LD (1992) Problems and strategies associated with longterm use of nematode resistant cultivars. J Nematol 24: 228-233

Young LD (1994) Changes in reproduction of a Heterodera glycines race 5 isolate cultured on 'Cordell' and 'Bedford' soybean. J Nematol 26(Suppl.):653-655

Yuhara I, Inagaki H (1963) Studies on the resistance of soybean plants to the soybean cyst nematode, Heterodera glycines II. The relations between the development of larvae invaded in the roots and the resistance of soybean varieties. Res Bull Hokkaido Natl Agric Exp Stn 80:94-102 (in Japanese with English summary) 